\title{
BMJ Open Rationale and design of a cross-sectional study to investigate and describe the chronotype of patients with type 2 diabetes and the effect on glycaemic control: the CODEC study
}

\author{
Emer M Brady, ${ }^{1}$ Andrew P Hall, ${ }^{2}$ Emma Baldry (D) , ${ }^{1}$ Sudesna Chatterjee, ${ }^{3}$ \\ Lois J Daniels, ${ }^{1}$ Charlotte Edwardson, ${ }^{3}$ Kamlesh Khunti (iD , ${ }^{3}$ Mubarak I Patel, ${ }^{3}$ \\ Joseph J Henson, ${ }^{3}$ Alex Rowlands, ${ }^{3}$ Alice C Smith, ${ }^{4}$ Thomas Yates, ${ }^{3}$ \\ Melanie J Davies ${ }^{3}$
}

To cite: Brady EM, Hall AP, Baldry E, et al. Rationale and design of a cross-sectional study to investigate and describe the chronotype of patients with type 2 diabetes and the effect on glycaemic control: the CODEC study. BMJ Open 2019;9:e027773. doi:10.1136/ bmjopen-2018-027773

- Prepublication history for this paper is available online. To view these files, please visit the journal online (http://dx.doi. org/10.1136/bmjopen-2018027773).

Received 06 November 2018 Revised 04 October 2019 Accepted 07 October 2019

Check for updates

(C) Author(s) (or their employer(s)) 2019. Re-use permitted under CC BY-NC. No commercial re-use. See rights and permissions. Published by BMJ.

For numbered affiliations see end of article.

Correspondence to

Dr Emer M Brady;

emb29@le.ac.uk

\section{ABSTRACT}

Introduction A person's chronotype is their entrained preference for sleep time within the 24 hours clock. It is described by the well-known concept of the 'lark' (early riser) and 'owl' (late sleeper). Evidence suggests that the 'owl' is metabolically disadvantaged due to the standard organisation of our society which favours the 'lark' and places physiological stresses on this chronotype. The aim of this study is to explore cardiometabolic health between the lark and owl in a population with an established metabolic condition - type 2 diabetes.

Methods This cross-sectional, multisite study aims to recruit 2247 participants from both secondary and primary care settings. The primary objective is to compare glycaemic control between late and early chronotypes. Secondary objectives include determining if latechronotype is associated with poorer cardiometabolic health and other lifestyle factors, including well-being, compared with early-chronotype; describing the prevalence of the five different chronotypes in this cohort and examining the trends in glycaemic control, cardiometabolic health, well-being and lifestyle factors across chronotype.

Analysis The primary outcome (glycated haemoglobin $(\mathrm{HbA} 1 \mathrm{c})$ ), linear regression analysis will compare $\mathrm{HbA1C}$ between early and late chronotypes, with and without adjustment for confounding variables. Chronotype will be modelled as a categorical variable with all five levels (from extreme-morning to extreme-late type), and as a continuous variable to calculate $p$ for trend across the five categories. A number of models will be created; unadjusted through to adjusted with age, sex, ethnicity, body mass index, duration of diabetes, family history of diabetes, current medication and dietary habits. All secondary outcomes will be analysed using the same method. Ethics Ethical approval from the West Midlands - Black Country Research Ethics Committee (16/WM/0457). Dissemination The results will be disseminated through publication in peer-reviewed medical journal, relevant medical/health conferences and a summary report sent to patients.

\section{Strengths and limitations of this study}

- A large phenotyped cohort of patients with established type2 diabetes mellitus for chronotype, levels of social jet lag and other parameters of sleep, physical activity and dietary behaviours and timing in addition to cardiometabolic profiling.

- Objective and subjective measures chronotype, levels of social jet lag and other parameters of sleep and physical activity in this large cohort collected.

- Cross-sectional study design therefore cannot establish causality of any identified associations.

Trialregistration number NCT02973412 (Pre-Results).

\section{INTRODUCTION}

The prevalence of diabetes mellitus has now reached over 4 million in the UK. ${ }^{1}$ Type 2 diabetes mellitus (T2DM) accounts for approximately $90 \%$ of all cases of diabetes and confers significant morbidity and mortality on patients and healthcare systems. Despite new therapies and management tools to improve quality of life and outcomes for patients with T2DM, cardiovascular disease (CVD) remains the greatest cause of mortality. New paradigms for characterising and treating these patients could enhance current T2DM management.

Recently, there has been considerable interest in the association between quantity and quality of sleep and circadian rhythms and the development of cardiometabolic disease especially metabolic syndrome, T2DM and CVD. ' A 'U'-shaped relationship exists between sleep duration and T2DM, obesity, CVD, hypertension and stroke. ${ }^{3-6} \mathrm{~A}$ meta-analysis of nearly 500000 individuals 
$(\sim 4 \%$ T2DM) identified a relative risk (RR) of $1.14(95 \%$ CI 1.03 to 1.26 ) for every additional hour of sleep and RR of 1.09 (95\% CI 1.04 to 1.15) with each hour of shorter sleep compared with 7 hours sleep per day for the development of T2DM. ${ }^{7}$ The negative impact of poor sleep on health is not widely known and subsequently, lifestyle choices, societal pressures and shift-work are rendering the population chronically sleep deprived and thus at increased risk of metabolic dysfunction. Indeed, the Sleep Council estimates that one-third of Britons sleep for just 5 to 6 hours per night which is well below the recommended 7 to 8 hours per night. ${ }^{8}$

Sleep is regulated, in part, by a homeostatic drive and is therefore unavoidable in humans (without sleep disorders). The circadian system, our internal clock, is also responsible for the regulation of sleep. Sleep is a multidimensional behaviour (and biological process) where we need to not only consider duration and quality but also timing. A person's sleep pattern, in relation to the 24 hour clock, that is, timing, is individual to them and referred to as their chronotype. We can quantify these individual differences in daily timing using a number of questionnaire based tools and objectively using for example accelerometers.

Five different chronotypes have been identified using the 'Morningness-Eveningness' Questionnaire that is, definite evening type, moderate evening type, intermediate, moderate morning type and definite morning type. ${ }^{9}$ The identification of these different chronotypes, which describes preferred circadian phases, into, at the two extremes, the 'lark' (early riser) and 'owl' (late sleeper) has led to further research confirming that 'owls' are at greater risk of cardiometabolic disease. ${ }^{10-12}$ The underlying causes have not been clearly defined but appear to be related to circadian misalignment causing chronic sleep deprivation and leading to dysregulation of metabolic, immune and hormonal processes that govern energy regulation and glycaemic control. ${ }^{13-15}$

The concept of 'social jet lag' has been developed to describe the deleterious effects of chronic sleep deprivation related to weekday occupational obligations on 'evening types' and weekend social demands on 'morning types'. ${ }^{16}$ These social pressures result in a disconnect or misalignment of entrained preference for sleep time and actual sleep time. Evidence of the deleterious effects of social jet lag is emerging. For example, a large epidemiological study in Germany has shown that social jet lag is associated with obesity. ${ }^{17} \mathrm{~A}$ recent systematic review reported associations between increasing levels of social jet lag and poorer mental health, epilepsy, cognitive impairment (eg, work and academic performance) and adverse endocrine profiles. ${ }^{18}$

Sleep is essential for human health however the mechanisms of sleep regulation are still not well established. There are limited data on objectively measured sleep and genetic variants that influence sleep traits such as chronotype ${ }^{19}$ Circadian rhythms have been shown not only to regulate sleep but several other physiological functions, including body temperature, physical activity, mood and cognition. These processes are controlled by circadian clock genes. Conversely the timing of behaviours such as sleep, exercise and food intake influence circadian rhythms, including clock gene expression. ${ }^{20} 21$

Further, individual components of phenotypic frailty, such as handgrip strength and walking pace, and composite scores such as Short Physical Performance Battery (SPPB), are established risk factors for mortality, morbidity and disability. ${ }^{2223}$ More generally, frailty, regardless of how it is defined, is associated with incident falls, difficulty with activities of daily living, disability, hospitalisation and death. ${ }^{24}$ While T2DM itself is associated with an elevated risk of mortality and healthcare utilisation, frailty magnifies these risks. ${ }^{25} \mathrm{It}$ is important to determine if there is an interaction between parameters of sleep, T2DM and individual components of frailty.

In this cross-sectional observational study, we therefore propose to extensively phenotype a sample of patients who have an existing chronic metabolic condition - T2DM, to determine the impact of chronotype and levels of social jet lag on their glycaemic control, insulin resistance, biochemical and inflammatory profiles and lifestyle behaviours using a variety of subjective and objective measures. This will build on the existing evidence base and permits exploration of the interrelationship between sleep behaviours, glycaemic control, cardiometabolic health and other lifestyle factors including physical fitness, wake-time activity and temporal eating habits in a multi-ethnic cohort with established T2DM

\section{METHODS AND ANALYSIS}

\section{Study design and population}

This is a cross-sectional, multisite observational study design. Two thousand two hundred and forty-seven patients with established T2DM ( $>6$ months), between the age of 18 to 75 years inclusive, without a current known sleep disorder (excluding obstructive sleep apnoea (OSA)) will be recruited from both primary and secondary care settings from four sites across the East Midlands, UK.

\section{Inclusion/exclusion criteria}

\section{Inclusion criteria}

- Participant is willing and able to give informed consent for participation in the study.

- Established T2DM ( $>6$ months since diagnosis).

- Male or female.

- Aged 18 to 75 years inclusive.

- Body mass index (BMI) less than or equal to $45 \mathrm{~kg} /$ $\mathrm{m}^{2}$ inclusive.

- No known sleep disorders except OSA.

- Glycated haemoglobin (HbA1c) up to and below $10 \%$ $(86 \mathrm{mmol} / \mathrm{mol})$.

- Type 1 diabetes.

- Good command of the English language. 
Exclusion criteria

- Participant is unwilling or unable to give informed consent.

- Anyone without a good command of the English language.

- Anyone $<18$ years of age and $>75$ years of age.

- HbAlc above $10 \%$ (86 mmol $/ \mathrm{mol})$.

- BMI greater than $45 \mathrm{~kg} / \mathrm{m}^{2}$.

- A regular cannabis user, that is, weekly use.

- Have a terminal illness.

- A known sleep disorder that is not OSA.

- Regular use ( $\geq$ weekly) of the following medications;

- Wakefulness promoting agents modafinil, amphetamine derivatives, methylphenidate.

- Sedatives including benzodiazepines, Z-drugs (zopiclone, zolpidem and zaleplon).

- Melatonin, including Circadin and melatonin analogues.

- Clonazepam and other drugs for nocturnal movement disorders.

\section{Sample size}

The primary objective is to determine if late-chronotype is associated with poorer glycaemic control compared with early-chronotype in this multi-ethnic cohort with established T2DM as assessed by HbA1c. In order to detect a minimum clinically significant difference in HbAlc of $0.4 \%$ between the two groups, assuming a SD of $1.8 \%,{ }^{78}$ power of $80 \%$, significance level of $5 \%$ and aiming to recruit approximately $50 \%$ morning type, $40 \%$ intermediate and $10 \%$ evening types, ${ }^{8} 1910$ participants were required. This has been inflated by $15 \%$ to include incomplete data set collection and withdrawal of consent. Therefore, a total of 2247 participants with T2DM are required for this study.

Secondary outcomes include; determining the prevalence of different chronotypes in this patient population, determine if late-chronotype is associated with poorer cardiometabolic health and other lifestyle factors, including physical fitness, well-being, compared with early-chronotype. We will describe the prevalence and phenotype of the five different chronotypes in a multiethnic UK population with established T2DM. Finally we will examine the trends in glycaemic control, cardiometabolic health, well-being and lifestyle factors across the five chronotype categories. All of the above will be explored within the context of social jet lag using a number of definitions for this construct. ${ }^{162627}$ Mid-sleep time (as an objective measure of chronotype) and other sleep parameters will be calculated from the accelerometer data.

\section{Study assessments}

This study involves a single data collection visit lasting approximately 1 to 1.5 hours. No data will be collected until informed consent has been provided by the participant. Where possible, data collection will be integrated with preplanned clinical appointments within outpatient departments. Where this is not possible, and for those recruited from primary care, specific study data collection visits will be arranged locally.

\section{Routine data}

The following data will be taken (where possible) from the patients notes following their routine appointment if they are recruited via either secondary or primary care, otherwise this data will be collected at their data collection appointment: date of birth, ethnicity, gender, HbAlc, random plasma glucose, full lipid profile, liver function tests, estimated glomerular filtration rate, creatinine, blood pressure, weight, BMI and waist circumference.

\section{ADDITIONAL STUDY-RELATED DATA TO BE COLLECTED Bioimpedance assessment}

We will conduct a bioimpedance assessment on participants to determine body composition. We will use a TANITA professional body composition monitor (SA 165A-095OU-3, Sino-American Electronics Co Ltd, Taiwan).

\section{Questionnaires}

A number of questionnaire will be administered to obtain self-reported measures of a number of outcomes detailed in the table 1 .

SINBAD and foot characteristics are only applicable to patients recruited in diabetes foot clinics. ${ }^{28}$ For patients recruited from diabetes foot clinics, the SINBAD score will be calculated. The SINBAD score is a classification system and score which compares outcomes of foot ulcer management. It is derived by six factors; Site, Ischaemia, Neuropathy, Bacterial infection, Area, Depth.

The participants offloading foot cast information will also be recorded. For this there are five categories:

- Below knee non-removable device/cast.

- Below knee removable device/cast.

- Non-removable foot device/cast.

- Removable foot device/cast.

- Usual orthotic/own shoes.

\section{Medical history}

Family history pertaining to cardiovascular disease, diabetes, obesity and depression will be obtained. Medical history will be collected and current concomitant medication in addition to smoking status and duration of diabetes. All those with an existing diagnosis of OSA will have the severity of their condition and compliance to therapy recorded. This will be collected in the case report form by the study clinician, research nurse or other appropriately trained member of the research team. Further, previous employment pertaining to previous night shift work for retired participants will be collected.

\section{Biochemistry}

Fasted venous blood samples (at least 4 hours of abstinence from food and anything other than water to drink) will be taken ( $9 \mathrm{~mL}$ EDTA) for the quantification of biological markers of metabolism and inflammation (eg, 
Table 1 Questionnaires used in CODEC study

\begin{tabular}{|c|c|c|c|c|}
\hline Outcome & Questionnaire & Items & $\begin{array}{l}\text { Time complete } \\
\text { (mins) }\end{array}$ & Score \\
\hline Chronotype $^{9}$ & $\begin{array}{l}\text { 'Morningness-Eveningness } \\
\text { Questionnaire }\end{array}$ & 19 & 5 & $\begin{array}{l}16-86 \text { and is categorised into three groups ranging } \\
\text { from evening }(16-53) \text { to morning }(64-86)\left({ }^{36}\right)\end{array}$ \\
\hline Physical activity ${ }^{37}$ & $\begin{array}{l}\text { Recent Physical Activity } \\
\text { Questionnaire }\end{array}$ & 57 & 5 & $\begin{array}{l}\text { Metabolic cost in metabolic equivalent tasks is } \\
\text { calculated - overall and for each section }\end{array}$ \\
\hline $\begin{array}{l}\text { Intake of pathogen } \\
\text { associated } \\
\text { molecular } \\
\text { patterns }^{38}\end{array}$ & $\begin{array}{l}\text { Pathogen Associated } \\
\text { Molecular Patterns } \\
\text { Questionnaire }\end{array}$ & 13 & 5 & $\begin{array}{l}0-5 \text { all questions except questions } 9 \text { and } 10(0-4) \text {. } \\
\text { Total provides the global score. }\end{array}$ \\
\hline Risk of OSA & $\begin{array}{l}\text { STOP-BANG } \\
\text { Questionnaire }\end{array}$ & 8 & 2 & $\begin{array}{l}\text { A score of } 5-8 \text { indicates a high probability of } \\
\text { moderate-severe OSA }\end{array}$ \\
\hline $\begin{array}{l}\text { Excessive daytime } \\
\text { sleepiness }^{39}\end{array}$ & Epworth Sleepiness Scale & 8 & 2 & $\begin{array}{l}\text { A total score between } 0-9 \text { indicative of no-daytime } \\
\text { sleepiness, } 10-12 \text { borderline, }>12 \text { describes } \\
\text { excessive daytime sleepiness }\end{array}$ \\
\hline
\end{tabular}

OSA, obstructive sleep apnoea.

high-sensitivity C-reactive protein (hs-CRP), interleukin 6 (IL-6), leptin, adiponectin) and a sample for C-peptide and insulin $(2.7 \mathrm{~mL}$ Lithium Heparin). These will be centrifuged and plasma aliquoted at source and stored at $-80^{\circ} \mathrm{C}$ at the Leicester Diabetes Centre (LDC). Samples will be analysed for hs-CRP, IL-6, leptin, adiponectin in batch, the remaining aliquots will be stored for future research, and this may be indefinite.

\section{PHYSICAL PERFORMANCE Handgrip strength}

Grip strength will be measured, using a digital hand-held dynamometer, three times on each side, with the elbow flexed at a right angle and the forearm in neutral position. The maximum of the readings generated is taken as the maximum grip strength.

\section{Short physical performance battery}

The SPPB measures balance, gait speed and ability to stand from a chair and has been shown to measure the risk of disability and mortality. ${ }^{29}$ It comprises of the tests outlined;

- Chair stands: The participant will start from a seated position on a hard, upright chair (such as a dining chair), with the feet flat on the floor and the knees bent at 90 degree. For the test, the time taken for the participant to stand up fully and then returning to sitting, without using the hands five times is measured.

- Standing balance: Tests in three progressive positions. If the participant is able to complete $10 \mathrm{~s}$ in the specified position then the starting position is progressed to the next stage:

- Feet together.

- Semi-tandem.
- Tandem.

- Gait speed: The time taken for the participant to walk 4 min on a level course. It is measured a second time after a short break.

\section{Sit-to-stand 60 test}

This test is similar to the chair standing test from the SPPB, but it measures how many times the participant can stand from a chair in 60 seconds. It is a surrogate measure of muscular endurance, therefore providing data on an additional marker of physical ability.

\section{SUBSTUDIES}

\section{Genetics}

From the viewpoint of elucidating physiological mechanisms and managing disease risk, it is important to examine the relationship between chronotype and social jet lag and clock gene expression. We will use the analysis of clock genes from a venous blood sample to examine the reciprocal impact of behaviour on circadian rhythm with a focus on clock genes and genes that increase the risk of diabetes and obesity. This will be a $9 \mathrm{~mL}$ EDTA sample of whole blood that will be transferred within 12 months of the study end date and stored at $-80^{\circ} \mathrm{C}$ in a Human Tissue Act (HTA) licensed facility for future ethically approved research. These samples may be stored indefinitely.

\section{Objectively measured physical activity and sleep}

Participants will be asked to wear an accelerometer (GENEActiv, Activinsights Ltd, Kimbolton, UK) on their non-dominant wrist to quantify habitual levels of physical activity and sleep. Participants will wear the GENEActiv accelerometer 24 hours/day for up to 8 days. Alongside this participants will fill out a wake and sleep log for the 
days they wear the device. The device will be fitted on the day of their appointment and they will be provided with a prepaid padded envelope addressed to the LDC in which to return the device and the wake/sleep log at the end of the assessment period.

\section{Diet and energy intake}

We will use a self-administered 3 day food record. Participants will be given verbal and written instructions on how to complete the record and be asked to start completing it from their first eating occasion the following day. Timing, frequency and content of eating occasions will be recorded. Dietary data will be entered into a specialist dietary analysis online tool (Nutritics). Software will be used to estimate total energy intake, and macronutrient intake. The temporal distribution of energy intake will be determined using the methodology described by Reutrakul et al. ${ }^{11}$

\section{Well-being}

Three questionnaires will be used: The Patient Health Questionnaire, ${ }^{30}$ the Diabetes Distress Scale-1 $17^{31-33}$ and the Self-Compassion Scale. ${ }^{34}$

\section{Description of statistical analysis}

The statistical analysis will be agreed prior to the data being made available to the study statistician(s). Participant characteristics will be summarised using either mean and $\mathrm{SD}$ or median and IQR for continuous variables as appropriate, and number and percentage for categorical variables. This will include the presentation of the number (percentage) of participants in each of the five different chronotype categories. Further stratification of the participant characteristics will be made according to the three categories of morning-evening types based on their Morningness-EveningnessQuestionnaire (MEQ) score. ${ }^{7}$ Analysis of variance or Kruskal-Wallis, as appropriate, will be used to analyse the relationship between MEQ category and the descriptive continuous variables, and $\mathrm{X}^{2}$ tests the relationship between MEQ category and descriptive categorical variables. Preliminary graphical and tabular examination of the data will be inspected for the correct statistical modelling assumptions.

For the primary outcome (HbAlc), linear regression analysis will compare $\mathrm{HbAlc}$ between early and late chronotypes, both with and without adjustment for confounding variables. The model will be fitted and reported with the MEQ category variable treated as a categorical variable, with early chronotype as the reference category. Additionally, the MEQ category variable will be modelled as a categorical variable with all five levels, and as a continuous variable to calculate $\mathrm{p}$ for trend across the five categories. Model 1 will be unadjusted. Model 2 will be adjusted for age, sex, ethnicity, BMI, duration of diabetes, family history of diabetes, whether currently administering anti-hypertensive medication and whether taking an oral or injectable therapy for their diabetes. ${ }^{35}$ Model 3 will be adjusted for all variables in model 2 plus dietary patterns. All secondary outcomes will be analysed using the same method as described above. Model assumptions will be checked and a more suitable model selected if these are not met. Subgroup analyses will be performed stratified by risk of OSA based on the STOPBANG questionnaire (high risk vs not).

Statistical analyses tests will be two-sided with a $5 \%$ significance level. The primary analyses will use a complete case analysis so only people with complete MEQ and HbAlc data will be included in the analyses; missing outcomes will not be replaced. Sensitivity analyses will include all recruited participants by fitting the same models, but with missing data replaced using multiple imputation or another suitable method.

\section{Patient and public involvement}

The development of the research question and outcome measures were presented and discussed at the Leicester Diabetes Centre Patient and Public Forum where it was agreed that the 'sleep' is not well understood or discussed in consultations with doctors at diabetes annual reviews (as is routine practice within England) or other such consultations. Specific contribution from patient and public involvement include the methods used to collect dietary information and the inclusion of the substudies as optional for participants because some people may feel overburdened with these measures. Patients were not involved in the recruitment to or conduct of the study. The results of the study will be disseminated to study participants by way of a newsletter and invitation to 'results' evening at the end of the study. At this event summary results will be presented and any conference posters will be displayed and provided in a 'results booklet'.

\section{Ethics and dissemination}

This study has been sponsored by the University of Leicester. Informed consent will be obtained before any data are collected. Each participant has the right to withdraw from the study at any time. In addition, the investigator may discontinue a participant from the study at any time if the investigator considers it necessary for any reason including; safety, study compliance or clinical reasons or if they withdraw their consent. The reason for withdrawal will be recorded in the case report form. However, participants will not need to provide a reason for their withdrawal if they do not wish not to do so. Unless the participant indicates otherwise, any data collected up to the point of consent withdrawal will be included in the final analysis

The results will be disseminated through publication in peer-reviewed medical journal, relevant medical/health conferences and a summary report sent to and presented to patients.

\section{Current study status}

This study is currently actively recruiting with 998 participants of the 2247 target recruited on submission of manuscript. Recruitment is planned to complete in June 2021. 
Author affiliations

${ }^{1}$ Leicester Diabetes Centre, University Hospitals of Leicester NHS Trust, Leicester, UK

${ }^{2}$ The Hanning Sleep Laboratory, University Hospitals of Leicester NHS Trust, Leicester, UK

${ }^{3}$ Diabetes Research Centre, University of Leicester, Leicester, UK

${ }^{4}$ John Walls Renal Unit, University Hospitals of Leicester NHS Trust, UK and Department of Infection, Immunity and Inflammation, University of Leicester, Leicester, UK

\section{Twitter Sudesna Chatterjee @DesChatterjee}

Acknowledgements The authors would like to thank and acknowledge the contribution of members of Leicester Diabetes Centre Patient and Public Forum in the design of this study.

Contributors EMB, APH, EB, SC, LD, CLE, KK, JH, AVR, AS, TY and MJD devised the project, the main conceptual ideas and proof outline. EMB wrote the manuscript with input from all authors. EMB and LD prepared and submitted research for local regulatory approvals. MIP contributed statistical support and will conduct all analysis.

Funding This work was supported by the Lifestyle theme of the National Institute of Health Research (NIHR) Leicester Biomedical Research Centre (BRC).

Competing interests None declared.

Patient consent for publication Not required.

Provenance and peer review Not commissioned; externally peer reviewed. Data availability statement № data are available.

Open access This is an open access article distributed in accordance with the Creative Commons Attribution Non Commercial (CC BY-NC 4.0) license, which permits others to distribute, remix, adapt, build upon this work non-commercially, and license their derivative works on different terms, provided the original work is properly cited, appropriate credit is given, any changes made indicated, and the use is non-commercial. See: http://creativecommons.org/licenses/by-nc/4.0/.

\section{ORCID iDs}

Emma Baldry http://orcid.org/0000-0002-9552-4143

Kamlesh Khunti http://orcid.org/0000-0003-2343-7099

\section{REFERENCES}

1 UK, D. Number of people with diabetes reaches over 4 million, 2016 Available: https://www.diabetes.org.uk/About_us/News/Number-ofpeople-with-diabetes-reaches-over-4-million/

2 Grandner MA, Alfonso-Miller P, Fernandez-Mendoza J, et al. Sleep: important considerations for the prevention of cardiovascular disease. Curr Opin Cardiol 2016;31:551-65.

3 Gangwisch JE, Heymsfield SB, Boden-Albala B, et al. Sleep duration as a risk factor for diabetes incidence in a large U.S. sample. Sleep 2007;30:1667-73.

4 Yaggi HK, Araujo AB, McKinlay JB. Sleep duration as a risk factor for the development of type 2 diabetes. Diabetes Care 2006;29:657-61.

5 Theorell-Haglöw J, Berglund L, Janson C, et al. Sleep duration and central obesity in women - differences between short sleepers and long sleepers. Sleep Med 2012;13:1079-85.

6 Leng Y, Cappuccio FP, Wainwright NWJ, et al. Sleep duration and risk of fatal and nonfatal stroke: a prospective study and metaanalysis. Neurology 2015;84:1072-9.

7 Shan Z, Ma H, Xie M, et al. Sleep duration and risk of type 2 diabetes: a meta-analysis of prospective studies. Diabetes Care 2015;38:529-37.

8 Sleepio. The great British sleep survey 2012; 2013.

9 Horne JA, Ostberg O. A self-assessment questionnaire to determine morningness-eveningness in human circadian rhythms. Int $J$ Chronobiol 1976;4:97-110.

10 Merikanto I, Lahti T, Puolijoki H, et al. Associations of chronotype and sleep with cardiovascular diseases and type 2 diabetes. Chronobiol Int 2013;30:470-7.

11 Reutrakul S, Hood MM, Crowley SJ, et al. Chronotype is independently associated with glycemic control in type 2 diabetes. Diabetes Care 2013;36:2523-9.

12 Vetter C, Devore EE, Ramin CA, et al. Mismatch of sleep and work timing and risk of type 2 diabetes. Diabetes Care 2015;38:1707-13.
13 Van Cauter E, Blackman JD, Roland D, et al. Modulation of glucose regulation and insulin secretion by circadian rhythmicity and sleep. $J$ Clin Invest 1991;88:934-42.

14 Spiegel K, Leproult R, Van Cauter E. Impact of sleep debt on metabolic and endocrine function. The Lancet 1999;354:1435-9.

15 Spiegel K, Tasali E, Penev P, et al. Brief communication: sleep curtailment in healthy young men is associated with decreased leptin levels, elevated ghrelin levels, and increased hunger and appetite. Ann Intern Med 2004;141:846-50.

16 Wittmann M, Dinich J, Merrow M, et al. Social jetlag: misalignment of biological and social time. Chronobiol Int 2006;23:497-509.

17 Roenneberg T, Allebrandt KV, Merrow M, et al. Social jetlag and obesity. Curr Biol 2012;22:939-43.

18 Castilhos Beauvalet J, Luísa Quiles C, Alves Braga de Oliveira M, et al. Social jetlag in health and behavioral research: a systematic review. ChronoPhysiology and Therapy 2017;7:19-31.

19 Jones SE, van Hees VT, Mazzotti DR, et al. Genetic studies of accelerometer-based sleep measures yield new insights into human sleep behaviour. Nat Commun 2019;10:1585.

20 Garaulet M, Madrid JA. Chronobiological aspects of nutrition, metabolic syndrome and obesity 2 . Adv Drug Deliv Rev 2010;62:967-78.

21 Takahashi M, Tahara Y, Tsubosaka M, et al. Chronotype and social jetlag influence human circadian clock gene expression. Sci Rep 2018;8:10152.

22 Yates T, Zaccardi F, Dhalwani NN, et al. Association of walking PACE and handgrip strength with all-cause, cardiovascular, and cancer mortality: a UK Biobank observational study. Eur Heart J 2017;38:3232-40.

23 Pavasini R, Guralnik J, Brown JC, et al. Short physical performance battery and all-cause mortality: systematic review and meta-analysis. BMC Med 2016;14:215.

24 Fried LP, Tangen CM, Walston J, et al. Frailty in older adults: evidence for a phenotype. J Gerontol A Biol Sci Med Sci 2001;56:M146-57.

25 Chao C-T, Wang J, Chien K-L, et al. Both pre-frailty and frailty increase healthcare utilization and adverse health outcomes in patients with type 2 diabetes mellitus. Cardiovasc Diabetol 2018;17:130.

26 Jankowski KS. Actual versus preferred sleep times as a proxy of biological time for social jet lag. Chronobiol Int 2017;34:1175-6.

27 Jankowski KS. Social jet lag: Sleep-corrected formula. Chronobiol Int 2017;34:531-5.

28 Ince P, Abbas ZG, Lutale JK, et al. Use of the Sinbad classification system and score in comparing outcome of foot ulcer management on three continents. Diabetes Care 2008;31:964-7.

29 Puthoff ML. Outcome measures in cardiopulmonary physical therapy: short physical performance battery. Cardiopulm Phys Ther $J$ 2008;19:17-22.

30 Kroenke K, Spitzer RL, Williams JBW. The PHQ-9. J Gen Intern Med 2001;16:606-13.

31 Fisher L, Glasgow RE, Mullan JT, et al. Development of a brief diabetes distress screening instrument. Ann Fam Med 2008;6:246-52.

32 Polonsky WHet al. Assessing psychosocial distress in diabetes. Development of the Diabetes Distress Scale 2005;28): :626-31.

33 Polonsky WH, Fisher L, Earles J, et al. Assessing psychosocial distress in diabetes: development of the diabetes distress scale. Diabetes Care 2005;28:626-31.

34 Neff KD. The development and validation of a scale to measure selfcompassion. Self and Identity 2003;2:223-50.

35 Gray LJ, Taub NA, Khunti K, et al. The Leicester risk assessment score for detecting undiagnosed type 2 diabetes and impaired glucose regulation for use in a multiethnic UK setting. Diabet Med 2010;27:887-95.

36 Taillard J, Philip P, Chastang J-F, et al. Validation of Horne and Ostberg morningness-eveningness questionnaire in a middle-aged population of French workers. J Biol Rhythms 2004;19:76-86.

37 Besson $\mathrm{H}$, Brage S, Jakes RW, et al. Estimating physical activity energy expenditure, sedentary time, and physical activity intensity by self-report in adults. Am J Clin Nutr 2010:91:106-14

38 Erridge $\mathrm{C}$. The capacity of foodstuffs to induce innate immune activation of human monocytes in vitro is dependent on food content of stimulants of Toll-like receptors 2 and 4 . Br J Nutr 2011;105:15-23.

39 Johns MW. A new method for measuring daytime sleepiness: the Epworth Sleepiness scale. Sleep 1991;14:540-5.

40 Ravani P, Kilb B, Bedi $\mathrm{H}$, et al. The Duke activity status index in patients with chronic kidney disease: a reliability study. Clin J Am Soc Nephrol 2012;7:573-80. 